\title{
Flipping the Visit: Impact of Blood Pressure Remeasurement after the Visit
}

\author{
Jeffrey D. Kravetz, $M D^{1,2} \odot$, Kara Cleveland, $R N^{2}$, and Scott Beauregard, $R N^{2}$ \\ 'Department of Internal Medicine, Yale University School of Medicine, New Haven, CT, USA; ${ }^{2}$ Veterans Affairs Connecticut Health Care System \\ (1 1ACSL), West Haven, CT, USA.
}

KEY WORDS: Hypertension; Blood pressure measurement; Overtreatment.

J Gen Intern Med 35(5): 1606-7

DOI: 10.1007/s11606-019-05359-y

(c) Society of General Internal Medicine (This is a U.S. government work and not under copyright protection in the U.S.; foreign copyright protection may apply) 2019

\section{INTRODUCTION}

Hypertension is one of the most common conditions managed in primary care and is a major risk factor for cardiovascular morbidity and mortality. With the release of the 2017 ACC/ AHA hypertension guidelines with new thresholds for diagnosis, the age-standardized prevalence of hypertension increased from $29 \%$ (JNC7 guidelines) to $45.5 \%$ in $2015-$ 2016. ${ }^{1,2}$ While hypertension remains one of the most impactful conditions primary care physicians manage, overtreatment can adversely affect patients. In a cohort of over 300,000 elderly patients newly treated for hypertension, there was a $43 \%$ increased risk of hip fracture occurring within 45 days following treatment initiation. ${ }^{3}$ In addition, low daytime systolic BP $(<128 \mathrm{mmHg})$ has been shown to be independently associated with greater progression of cognitive decline in a cohort of elderly patients with baseline cognitive impairment. ${ }^{4}$ Accurate assessment of BP is imperative to avoid overtreatment, especially in the elderly population. It is well known that many factors affect BP measurement, including talking, smoking, mental stress, and measurement in the inappropriate position and over clothing. ${ }^{5}$ Many of these factors exist just prior to an office visit and can be eliminated if the BP is assessed following the visit. We undertook this quality improvement initiative to determine the impact of BP reassessment after a clinic visit in a select population of male veterans.

\section{METHODS}

The West Haven Veterans Affairs medical center takes care of approximately 14,000 veterans, half of whom receive primary care in the Firm A clinic. In this clinic, vital signs are checked

Received July 22, 2019

Revised August 8, 2019

Accepted September 12, 2019

Published online October 24, 2019 prior to the visit and entered into the electronic medical record. The primary care provider can repeat the BP if the initial BP is elevated. For patients with elevated repeat BPs (defined as systolic $>140$ ), we established a protocol whereby these patients could have their BP rechecked after the visit. The repeat BPs were performed in the appropriate position with the correct cuff size over bare skin while using an electronic monitor set on a 5min timer. The patient was asked to empty his/her bladder and abstain from using a cell phone during the repeat check. Repeat BPs were performed in a quiet room with the lights dimmed. The repeat BP was recorded and compared with the initial BP.

\section{RESULTS}

Fifty-eight patients had repeat BPs obtained following their primary care visit. All patients were male with a mean age of 72.3 (range 34-96). Forty-three patients were taking antihypertensive medications prior to their visit, with a median of 2 medications per patient. The mean and median BPs prior to the visit were $154.7 / 75.8 \mathrm{mmHg}$ and $150.5 / 75 \mathrm{mmHg}$. The repeat BP mean and median decreased by $17.8 / 2.3 \mathrm{mmHg}$ and $14 /$ $2.5 \mathrm{mmHg}$ respectively. (Table 1) The repeat systolic BP was $<140 \mathrm{mmHg}$ in 37 patients, while the repeat diastolic BP was $<90 \mathrm{mmHg}$ in 57 patients.

\section{DISCUSSION}

The first step to appropriately manage hypertension in the primary care setting is to accurately measure a patient's BP. The standard routine of checking BPs immediately upon checking into a clinic has many potential pitfalls that can unintentionally raise a patient's BP, including poor patient positioning, lack of appropriate rest after arrival, smoking a cigarette prior to the visit, mental stress, and talking during check in. We believe that the repeat BP by a clinician is also fraught with problems, including the stress of the actual visit and not allowing for enough time to rest. Treating a patient for hypertension based on this one time reading might lead to overtreatment and potentially harmful outcomes.

Our quality improvement study has shown that a simple, easy-to-implement repeat BP in an appropriate position and quiet room has the ability to screen out falsely elevated BPs in a large proportion of patients, whether or not they are 
Table 1 Blood Pressure Before and After the Primary Care Visit ( $S B P$ Systolic BP in $\mathrm{mmHg}, D B P$ Diastolic BP in $\mathrm{mm} \mathrm{Hg}$ )

\begin{tabular}{|c|c|c|c|c|c|c|}
\hline & \multicolumn{2}{|l|}{ Before } & \multicolumn{2}{|l|}{ After } & \multicolumn{2}{|c|}{ Change } \\
\hline & SBP & DBP & SBP & DBP & SBP & DBP \\
\hline Mean & 154.7 & 75.8 & 136.9 & 73.5 & -17.8 & -2.3 \\
\hline Median & 150.5 & 75 & 137 & 74 & -14 & -2.5 \\
\hline St Dev & 12.8 & 9.3 & 12.5 & 8.8 & 11.4 & 7.3 \\
\hline
\end{tabular}

prescribed antihypertensive medications. The mean reduction of $\mathrm{BP}$ of $17.8 / 2.3 \mathrm{mmHg}$ is consistent with a previously published repeat BP study which involved automated BPs over a 30-min period, but on a different day in clinic. ${ }^{6}$ Our study can easily be implemented in a busy clinical practice without the requirement of a repeat visit or extra staffing and has the possibility of reducing overtreatment of some patients.

Corresponding Author: Jeffrey D. Kravetz, MD; Veterans Affairs Connecticut Health Care System (11ACSL), West Haven, CT, USA (e-mail: Jeffrey.Kravetz@va.gov).

\section{Compliance with Ethical Standards:}

Conflict of Interest: None of the authors has any conflicts of interest.

\section{REFERENCES}

1. Whelton PK, et al. 2017 ACC/AHA/AAPA/ABC/ACPM/AGS/APhA/ ASH/ASPC/NMA/PCNA Guideline for the prevention, detection, evaluation, and management of high blood pressure in adults: a report of the American College of Cardiology/American Heart Association Task Force on Clinical Practice Guidelines. Hypertension. 2018; 71:e13-e115.

2. Dorans KS, et al. Trends in Prevalence and Control of Hypertension According to the 2017 American College of Cardiology/American Heart Association (ACC/AHA) Guideline. J Am Heart Assoc. 2018; 7:e008888. DOI:https://doi.org/10.1161/JAHA.118.008888.

3. Butt DA, et al. The risk of hip fracture after initiating antihypertensive drugs in the elderly. Arch Intern Med. 2012;172(22):1739-1744.

4. Mossello E, et al. Effects of low blood pressure in cognitively impaired elderly patients treated with antihypertensive drugs. JAMA Intern Me. 2015;175(4):578-585.

5. Handler $\mathbf{J}$. The importance of accurate blood pressure measurement. Perm J. 2009; 13:51-54.

6. Bos MJ, Buis $\mathbf{S}$. Thirty-minute office blood pressure monitoring in primary care. Ann Fam Med. 2017;15:120-123.

Publisher's Note Springer Nature remains neutral with regard to jurisdictional claims in published maps and institutional affiliations. 